\title{
EFFECT OF BRETHING EXERCISE ON REDUCING PAIN ASSOCIATED WITH DRESSING AMONG HOSPITALIZED BURNED PATIENTS
}

\author{
Prof. Ahmed Abdelgilil Khalil'; ${ }^{1}$ Dr. Eman Saleh Shahin²; Dr. Amal Bakr Abo El-ata ${ }^{3}$;
} Abeer Mohamed NaserEldin Yousef Saleh ${ }^{4}$

Professor of Cosmetic Surgery - Faculty of Medicine - Mansoura University ${ }^{l}$; Assistant Professor of Medical Surgical Nursing - Faculty of Nursing - Port Said University ${ }^{2}$; Assistant Professor of Medical Surgical Nursing-Faculty of Nursing - Port Said University³; BSC Nursing - Zagazig University ${ }^{4}$

\begin{abstract}
Background: Burns can lead to severe mental and emotional distress. Aim: Assess effect of breathing exercise on reducing pain associated with dressing among hospitalized burned patients. Subjects and method: Design: A Quasi-experimental research design was utilized; the study was carried out. Setting: study performed in center of burn and beauty center at Mansoura University Hospital. Subjects: carried out on 73 burnt patients attended to study setting. Tools: Two tools were used to collect data of the current study. Tool (I) Patient's questionnaire which consist of three parts; Part 1: Socio demographic data Part 2: Patient burn history: Part 3: The Burns Specific Pain Anxiety Scale and Tool (II): Observational Checklist which consist of three parts; Part 1: "Burn Parameters" Part 2: University of Alabama at Birmingham UAB3 Relaxed Behaviors Checklist. Results: There were an improvement in results of burn specific pain anxiety scales score, UAB Pain Behavior Scale and relaxed behavior after application of breathing exercise. The study Conclusion: that using of breathing exercise are very important to reduce pain and anxiety associated with burn dressing change for hospitalized burned patients. Recommendations: Training programs should be applied for patients on breathing exercise as daily routine nursing care to reduce pain and anxiety associated with dressing change.
\end{abstract}

Key words: Breathing exercise, Hospitalized burned patients, Pain Associated with dressing 


\section{INTRODUCTION}

Burns are the most extensive forms of soft tissue injuries occasionally resulting in extensive and deep wounds and death. Burns can lead to severe mental and emotional distress, because of excessive scarring and skin contractures (Oryan, Alemzadeh \& Moshiri, 2017). Burns are devastating to the individual and society, representing a huge biomedical burden. Improved education in burns has however ignited a revolution in high-income countriesburn mortality is reducing. Education in burns is far-reaching (Tevlin, Dillon \& Clover, 2017).

When considering burns dressings, it is useful to remember the theory of Jackson's burn wound model. The inner zone of a burn is the zone of coagulation. This area is dead and nothing any clinician can do will bring it back to life. The outer zone of the burn is called the zone of hyperaemia. This is a reactive zone of inflammation in response to the injury, which can occur with non-burn injuries such as trauma, and will return to normal within hours of the injury. The middle zone of the burn is called the zone of stasis, which is the target of good burns care (Douglas \&Wood, 2017).

Wound care in burn patients is associated with severe anxiety that is characterized by feeling of fear and prediction of burn dressing pain. Assessment and treatment of pain anxiety are essential elements of pain care and management in burn patient (BozorgNejad, 2018) Moreover, stress and pain can result from wound care itself. Dressing changes are often painful for patients, doctor's appointments and wound care visits can be taxing financially and time consuming, thus inducing anxiety and (Hughes, MacQuhae, Rakosi, Herskovitz, \& Kirsner, 2016).

Burn pain varies greatly from patient to patient, shows substantial fluctuation over the hospitalization course, and can be unpredictable due to the complex interaction of anatomic, physiologic, psychosocial, and premorbid behavior issues Burn patients typically report pain as being severe or excruciating, despite receiving opioid analgesics (Alaa Eldin, Mohamed, \& Ragab, 2015)

Nurses must also be skilled in pain assessment and employing strategies to minimize the pain experience of the patient because some patients may experience both physiologic and/or psychological pain related to dressing changes and wound care (Lynn, 2015). 
Measures that can be used for reducing anxiety and pain during painful dressing procedures suggested by (Culleiton and Simko, 2013) these measures include relaxation techniques, distraction, guided imagery, hypnosis and therapeutic touch. A simple breathing relaxation technique is a simplest behavioral intervention to manage pain and anxiety, because it involves no risk, easy and quick to learn, equipment does not need to be purchased and it can be employed immediately by the often exhausted and ill patient (Park, Oh, \& Kim, 2013).

However, the information about the effects of breathing technique on pain and anxiety levels for burn patients during dressing changes is limited. Muscle tension in burned parts of the body can further increase pain during wound care. In other words, even a simple technique such as progressive muscle relaxation can be inappropriate for patients with burns. More benefit for these patients with burns is expected from the use of breathing techniques in order to gain control over their pain (De Jong \& Gamel, 2015).

\section{Significance of the study:}

Most wound care procedures for example cleaning, dressing and physiotherapy are very painful hence severe pain may increase risk of developing anxiety and minimize patient's participation in burn rehabilitation hence increasing hospital stay and morbidity (Park, Shin, Kim, Song, \& Peck, 2009).

\section{AIM OF THE STUDY:}

The aim of the present study is to assess effect of breathing exercise on reducing pain associated with dressing among hospitalized burned patients

\section{Research question:}

What is the effect of breathing exercise on reducing pain associated with dressing among hospitalized burned patients?

\section{SUBJECTS AND METHOD:}

\section{I: TECHNICAL DESIGN}

\section{Research design:}

A Quasi-experimental research design (pretest, posttest) was utilized. 


\section{Setting:}

The study was conducted in center of burn and beauty at Mansoura University Hospital. It is a building consist of six floors, patients is taken from first and second floor, first floor consist of two rooms; each room with 4 beds and three room each one with 2 beds, second floor consist of two room; each one consist of two beds and one room with 3 beds, The center works 48 beds, intensive care unit with five beds in the second floor.

\section{Patients and sampling technique}

A convenient sample was used, it consists of seventy three adult patients newly admitted to burn unit participated in the current study, patients were selected according to the following inclusion criteria (WHO, 2011).

- Alert patients

- $2^{\text {nd }}$ degree of burns; $15 \%-25 \%$. of (TBSA)

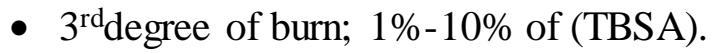

\section{Exclusion criteria}

Patient with associated trauma as fracture or poly trauma and inhalation injuries.

\section{Tools of data collection:}

Two Tools were used to gather data

\section{Tool (I): Patient's questionnaire}

It was adopted from (Morsy and Alam, 2014), and used to collect information about patient's past medical history, burn history and burn specific pain anxiety scale. It consists of two parts:

Part (1): Socio demographic characteristics; It reports data about patients age, sex, conjugal status, instructive level, occupation, family salary, and information of date.

Part (2): Patient's history: It records the reason for patient length of contact with consumed operators and medical aid of consume utilized by the patients or relatives, for example water use, cream, or any substance.

Part (3): The "consume particular torment tension scale: It was developed by (Asgharipour, Shariati \& Borhani, 2017).

Tool (II): Observational Checklist: 
It was made out of three section to survey consume parameters torment practices scale and loosened up behavior and it incorporate, and it include the following parts.

Part (1): "Burn Parameters" It incorporates things identified with sit of consume damage, percent of burn, color of burn and degree of consume damage.

\section{Part (2): " University of Alabama at Birmingham UAB3 Relaxed Behaviors}

Checklist." This scale was created to survey seriousness of torment through perception of agony related practice for example; verbal complaints, non-verbal response, facial grimaces, standing posture, mobility, body dialect utilization of noticeable support, stationary movement, and torment pharmaceutical (Park, et al., 2013).

Part (3): Relaxed Behaviors Checklist: this part evaluate patients' loose and unrelaxed conduct through perception of verbal sound, body position, head motion, eyes, mouth and imperative signs (blood pressure, pulse and breath). Done take score 1 and not done take zero

\section{II: OPERATIONAL DESIGN}

The operational design includes Preparatory phase, Validity, Reliability, Pilot Study, and field of work.

\section{A- Preparatory Phase:}

It will includes reviewing of recent related literature, different studies, theoretical knowledge of various aspects of problem using books, articles, internet, periodic magazines, references and the official research websites as (Cinhol, pubmed, ebesco and cochrane library).

\section{B- Content Validity:}

It was asserted by nine jury in the field of restorative careful nursing, Critical mind nursing (five experts), burn surgery and cosmetic physicians (four experts) .The questionnaires were modified according to the experts, comments and recommendations.

\section{C - Reliability:}

The consume particular agony burn specific anxiety scale is exceptional and substantial pointer of torment-related anxiety encompassing mind in hospitalized patients. The dependability of this was high 0.94. The unwavering quality of college of Alabama at Birmingham used in this study was acknowledged and it was found 0.95.

\section{D -Pilot Study:}

A pilot study was conducted on $10 \%$ (8) of patients from burn unit in order to test the clarity and feasibility of different items of the tools .Modification rephrasing and some 
additional terms done by the researcher before study according to the experience gained from pilot study . Data obtained from those patients will be excluded.

\section{Filed work:}

The actual fieldwork was carried out over the period from the beginning of July 2016 up to the end of December 2016 for data collection. Before data collection the process and purpose of the study were explained to each patient. The researcher observed the patients by using burn parameters, university of Alabama at Birmingham" UAB pain behavior scale and relaxed behaviors checklist before and after breathing exercise use during dressing by indirect observation guided by observation checklists in the morning, afternoon and night shifts.

\section{III: ADMINISTRATIVE DESIGN}

An official letter was issued from the Faculty of Nursing, Port Said University to the director of center of burn and beauty at Mansoura University Hospital to obtain their permission to conduct the current study. The researcher explained the aim of the present study to the director of burn unit to take his permission to carry out this study.

\section{The study will be carry on three phases:}

\section{Assessment stage (Pre mediation stage)}

- Each of the 73 grown-up patients who satisfy incorporation criteria and will take part in the present study, the scientist watched the patient amid this stage (first week) in second day of admission to the consume unit.

- Each concentrated patient was met separately around 30 minutes to gather the fundamental information identified with device I. The analyst likewise utilized Instrument II: perception agenda which partitioned into three sections Section 1: consume parameter to evaluate site of consume damage, add up to body surface zone and profundity of consume damage, Part II: UAB torment scale conduct to survey torment related practices and Part III: loosened up practices to survey patients, loose and unrelaxed practices through perception of verbal sound, body position, head movement, eyes, mouth and estimating indispensable signs (circulatory strain, heartbeat and breath).

Also, the researcher assessed available places, time, equipment, supplies, and instructional material for conduction of training program. 


\section{2- Intervention stage:}

Breathing exercise was connected (during dressing) at this stage for resulting two weeks ( $2^{\text {nd }}$ week and $3^{\text {rd }}$ week), it applied in four sessions divided as the following:

1- First session: (Early on session) introduction and clarification of unwinding technique and benefit from utilization of these technique.

2- Second session: clarify of component of breathing, advantage of loosened up breathing exercise and preparing on relaxing exercise.

3- Third session: preparing on profound loose and record relaxing exercise.

4- Fourth session: revision on previously trained breathing exercise.

\section{3- Post intervention phase:}

By using tool (II) part (III) relaxed behaviors checklist, the researcher used it to assess the effect breathing exercise on reducing pain during dressing.

1- Breathing hone sessions were helped out with every patient independently through meeting that led for 20-30 minutes before dressing changes.

2- The scientists prepared the patients of breathing exercise independently in every session through demonstration, re-show and pretending.

3- The patients performed previously taught breathing exercises in presence of researcher during dressing change with simple instruction provided by researcher during dressing for two weeks.

\section{Ethical Consideration:}

Ensuring the confidentiality of the information collected and anonymity is guaranteed. Consent was obtained from patients after explanation of the purpose of the study confidentiality and privacy was assured. The objective and the aim of study were explained to participants. The researcher maintain an anonymity of subjects, were allowed to choose to participate or not and they have the right to withdrawal from the study at any time without penalty.

\section{IV: STATISTICAL DESIGN}

Data were extracted from the interview questionnaire and computerized in Microsoft Excel 2010. Analysis was undertaken using SPSS (statistical package for social science) version 22.0.The data were explored. Qualitative data were described using number and percent. Descriptive statistics were used for quantitative variables as mean and standard deviation. Paired t-test was used to compare between the pre-test and post-test; ANOVA test was used to compare between more than two variables. Qualitative variables were compared 
using chi square test $(\mathrm{X} 2)$. The p-value is the degree of significance that considered when the $\mathrm{p}$-value $\leq 0.05$.

\section{RESULTS:}

Table (1): It revealed that the highest percent of studied patients $(38.4 \%)$ their ages ranged from 20 to less than 30 years old with a mean age $28.79 \pm 8.95$ years. $(63 \%)$ were males. (56.2\%) were living in rural area. Regarding educational level of studied patients (47.9\%) have basic education. Finally, $(41.1 \%)$ of studied subjects working in manual work, married and from rural $(60.3 \%, 56.2 \%$ respectively.

Table (2): There were statistically significant deference between Burns specific pain anxiety scale score before and after use of breathing exercises during dressing exchange among burned patients with $\mathrm{p}$-value $=0.000$

Table (3): shows that there were statistically significant difference in relation to total burn specific pain anxiety scale before and after using breathing exercise during exchange dressing among burned patients in second and third week at $\mathrm{p}=0.000$

Table (4): It was observed that there were statistically significant difference in relation to $\mathrm{UAB}$ pain scale behavior before and after using breathing exercise during exchange dressing among burned patients in second and third week with $\mathrm{p}=0.000$.

Table (5): This table demonstrated that there were a statistical significance difference between second, and third week pre and post using breathing exercise regarding relaxed behavior with $\mathrm{p}(0.001$ and 0.002$)$ respectively.

Table (6):It was observed that total systolic blood pressure Mean \pm SD in the first week was $(149.47 \pm 11.32)$ during exchange of dressing, at the second weeks before and after use of breathing exercises during exchange dressing total mean value was $(153.13 \pm 11.80)$, $(153.13 \pm 11.80)$ respectively. At the third weeks the mean value was $(152.66 \pm 10.56)$, (146.99 \pm 10.43$)$ respectively before and after use of breathing exercises during exchange dressing. There was statistically significant differences between pulse measurement in days of the second and third week with $\mathrm{p}=0.000$. 
In connection to diastolic circulatory strain at first weeks, add up to mean was (83.72 \pm 4.94$)$.While at second weeks $(83.26 \pm 5.75)$ and (79.84 \pm 5.49$)$ before and after use of breathing exercises during exchange dressing. At the third weeks the mean value was (152.66 \pm 10.56$)$, $(146.99 \pm 10.43)$ respectively before and after use of breathing exercises during exchange dressing. There was statistically significant differences between pulse measurement in days of the second and third week with $\mathrm{p}=0.000$.

Also to respiratory rate at first weeks the mean value an incentive at first weeks total mean value was $(25.96 \pm 1.71)$.In the second weeks total mean was $(25.66 \pm 1.81),(24.63 \pm 1.30)$ respectively before and after use of breathing exercises during exchange dressing. In the third weeks total mean was $(25.47 \pm 1.83),(24.59 \pm 1.34)$ respectively before and after use of breathing exercises during exchange dressing. There was statistically significant differences between pulse measurement in days of the second and third week with $\mathrm{p}=0.000$.

As to beat rate it was accounted for that aggregate mean esteem was $(94.49 \pm 10.24)$ in the first week. In the second week total mean value was $(94.49 \pm 10.2),(90.40 \pm 8.26)$ respectively before and after use of breathing exercises during exchange dressing. In the third weeks total mean was $(94.49 \pm 10.24$ and90.40 \pm 8.26$)$ respectively before and after use of breathing exercises during exchange dressing. There was statistically significant differences between pulse measurement in days of the second and third week with $p=0.000$. 
Table (1): Socio-demographic characteristics of the studied burned patients $(n=73)$

\begin{tabular}{|c|c|c|}
\hline Characteristics & No & $\%$ \\
\hline Age (year) & & \\
\hline less than 20 (18 years) & 17 & 23.3 \\
\hline $20<30$ & 28 & 38.4 \\
\hline $30<40$ & 19 & 26.0 \\
\hline $40<50$ & 8 & 11.0 \\
\hline More than 50 & 1 & 1.4 \\
\hline Min - Max & \multirow{2}{*}{\multicolumn{2}{|c|}{$\begin{array}{c}15-53 \\
28.79 \pm 8.95\end{array}$}} \\
\hline Mean \pm SD & & \\
\hline \multicolumn{3}{|l|}{ Gender } \\
\hline Male & 46 & 63.0 \\
\hline Female & 27 & 37.0 \\
\hline \multicolumn{3}{|l|}{ Marital status } \\
\hline Married & 44 & 60.3 \\
\hline Divorced & 5 & 6.8 \\
\hline Single & 21 & 28.8 \\
\hline Widow & 3 & 4.1 \\
\hline \multicolumn{3}{|l|}{ Residence } \\
\hline Rural & 41 & 56.2 \\
\hline Urban & 32 & 43.8 \\
\hline \multicolumn{3}{|l|}{ Educational level } \\
\hline Illiterate & 12 & 16.4 \\
\hline Read and write & 5 & 6.8 \\
\hline Basic education & 35 & 47.9 \\
\hline Intermediate education & 4 & 5.5 \\
\hline Secondary education & 10 & 13.7 \\
\hline Post graduate & 7 & 9.6 \\
\hline \multicolumn{3}{|l|}{ Occupation } \\
\hline Manual work & 30 & 41.1 \\
\hline Employee & 6 & 8.2 \\
\hline Technical work & 11 & 15.1 \\
\hline Housewife & 17 & 23.3 \\
\hline Student & 1 & 1.4 \\
\hline Not work & 8 & 11.0 \\
\hline
\end{tabular}


Table (2): Continue... Burns specific pain anxiety scale score s before and after use of breathing exercises during dressing exchange among burned patients $(n=73)$.

\begin{tabular}{|c|c|c|c|c|c|c|c|c|c|c|c|c|}
\hline \multirow[t]{3}{*}{ Variables } & \multirow{2}{*}{\multicolumn{2}{|c|}{$\begin{array}{c}\begin{array}{c}\text { Dressing in } \\
\text { the first } \\
\text { week }\end{array} \\
\begin{array}{c}\text { Pain } \\
\text { assessment }\end{array}\end{array}$}} & \multicolumn{4}{|c|}{$\begin{array}{l}\text { Dressing in the second- } \\
\text { week }\end{array}$} & \multirow[t]{3}{*}{ Sign } & \multicolumn{4}{|c|}{ Dressing in the third-week } & \multirow[t]{3}{*}{ Sign } \\
\hline & & & \multicolumn{2}{|c|}{$\begin{array}{c}\text { Pre- } \\
\text { breathing } \\
\text { exercise }\end{array}$} & \multicolumn{2}{|c|}{$\begin{array}{c}\text { Post- } \\
\text { breathing } \\
\text { exercise }\end{array}$} & & \multicolumn{2}{|c|}{$\begin{array}{l}\text { Pre- breathing } \\
\text { exercise }\end{array}$} & \multicolumn{2}{|c|}{$\begin{array}{c}\text { Post- } \\
\text { breathing } \\
\text { exercise }\end{array}$} & \\
\hline & No & $\%$ & No & $\%$ & No & $\%$ & & No & $\%$ & No & $\%$ & \\
\hline $\begin{array}{l}\text { Anxiety } \\
\text { level six day } \\
\text { Mild } \\
\text { Moderate } \\
\text { Severe }\end{array}$ & $\begin{array}{c}0 \\
9 \\
64\end{array}$ & $\begin{array}{c}0.0 \\
12.3 \\
87.7\end{array}$ & $\begin{array}{c}0 \\
68 \\
5\end{array}$ & $\begin{array}{c}0.0 \\
93.2 \\
6.8\end{array}$ & $\begin{array}{c}0 \\
68 \\
5\end{array}$ & $\begin{array}{c}0.0 \\
93.2 \\
6.8\end{array}$ & $\begin{array}{l}\mathrm{F}=106.152 \\
\mathrm{p}=0.000 * *\end{array}$ & $\begin{array}{l}10 \\
50 \\
13\end{array}$ & $\begin{array}{l}13.7 \\
68.5 \\
17.8\end{array}$ & $\begin{array}{l}35 \\
25 \\
13\end{array}$ & $\begin{array}{l}47.9 \\
34.2 \\
17.8\end{array}$ & $\begin{array}{l}\mathrm{F}=106.152 \\
\mathrm{p}=0.000^{*} *\end{array}$ \\
\hline $\begin{array}{l}\text { Anxiety } \\
\text { level seven } \\
\text { day } \\
\text { Mild } \\
\text { Moderate } \\
\text { Severe } \\
\end{array}$ & $\begin{array}{c}0 \\
10 \\
63\end{array}$ & $\begin{array}{c}0.0 \\
13.7 \\
86.3\end{array}$ & $\begin{array}{c}0 \\
68 \\
5\end{array}$ & $\begin{array}{c}0.0 \\
93.2 \\
6.8\end{array}$ & $\begin{array}{c}0 \\
73 \\
0\end{array}$ & $\begin{array}{c}0.0 \\
100.0 \\
0.0\end{array}$ & $\begin{array}{l}\mathrm{F}=95.018 \\
\mathrm{p}=0.000 * *\end{array}$ & $\begin{array}{l}10 \\
50 \\
13\end{array}$ & $\begin{array}{l}13.7 \\
68.5 \\
17.8\end{array}$ & $\begin{array}{l}44 \\
16 \\
13\end{array}$ & $\begin{array}{l}60.3 \\
21.9 \\
17.8\end{array}$ & $\begin{array}{l}\mathrm{F}=129.393 \\
\mathrm{p}=0.000^{*} *\end{array}$ \\
\hline
\end{tabular}

Table (3): Total burns specific pain anxiety scale before and after use of breathing exercises during dressing exchange among burned patients $(n=73)$.

\begin{tabular}{|c|c|c|c|c|c|c|c|c|c|c|c|c|}
\hline \multirow[t]{3}{*}{ Variables } & \multirow{2}{*}{\multicolumn{2}{|c|}{\begin{tabular}{|c|}
$\begin{array}{c}\text { Dressing } \\
\text { in the } \\
\text { First week }\end{array}$ \\
$\begin{array}{c}\text { Pain } \\
\text { assessment }\end{array}$ \\
\end{tabular}}} & \multicolumn{4}{|c|}{$\begin{array}{l}\text { Dressing in the } \\
\text { Second week }\end{array}$} & \multirow[t]{3}{*}{ Sign } & \multicolumn{4}{|c|}{$\begin{array}{l}\text { Dressing in the Third } \\
\text { week }\end{array}$} & \multirow[t]{3}{*}{ Sign } \\
\hline & & & \multicolumn{2}{|c|}{$\begin{array}{c}\text { Pre } \\
\text { breathing } \\
\text { exercise }\end{array}$} & \multicolumn{2}{|c|}{$\begin{array}{c}\text { Post- } \\
\text { breathing } \\
\text { exercise }\end{array}$} & & \multicolumn{2}{|c|}{$\begin{array}{c}\text { Pre } \\
\text { breathing } \\
\text { exercise }\end{array}$} & \multicolumn{2}{|c|}{$\begin{array}{c}\text { Post- } \\
\text { breathing } \\
\text { exercise }\end{array}$} & \\
\hline & No & $\%$ & No & $\%$ & No & $\%$ & & No & $\%$ & No & $\%$ & \\
\hline $\begin{array}{l}\text { Total } \\
\text { anxiety } \\
\text { level } \\
\text { Mild } \\
\text { Moderate } \\
\text { Severe } \\
\end{array}$ & $\begin{array}{c}0 \\
0 \\
73\end{array}$ & $\begin{array}{c}0.0 \\
0.0 \\
100.0\end{array}$ & $\begin{array}{c}0 \\
16 \\
57\end{array}$ & $\begin{array}{c}0.0 \\
21.9 \\
78.1\end{array}$ & $\begin{array}{c}0 \\
63 \\
10\end{array}$ & $\begin{array}{c}0.0 \\
86.3 \\
13.7\end{array}$ & $\begin{array}{c}\mathrm{F}= \\
112.545 \\
\mathrm{p}= \\
0.000^{* *}\end{array}$ & $\begin{array}{l}10 \\
50 \\
13\end{array}$ & $\begin{array}{l}13.7 \\
68.5 \\
17.8\end{array}$ & $\begin{array}{l}33 \\
27 \\
13\end{array}$ & $\begin{array}{l}45.2 \\
37.0 \\
17.8\end{array}$ & $\begin{array}{c}\mathrm{F}= \\
90.452 \\
\mathrm{p}= \\
0.000^{* *}\end{array}$ \\
\hline
\end{tabular}


Table (4): UAB Pain Behavior Scale among the studied burned patients $(n=73)$

\begin{tabular}{|c|c|c|c|c|c|c|c|c|c|c|c|c|}
\hline \multirow{3}{*}{ Variables } & \multirow{2}{*}{\multicolumn{2}{|c|}{$\begin{array}{c}\begin{array}{c}\text { Dressing in } \\
\text { the first } \\
\text { week }\end{array} \\
\text { Pain } \\
\text { assessment }\end{array}$}} & \multicolumn{4}{|c|}{$\begin{array}{l}\text { Dressing in the second } \\
\text { week }\end{array}$} & \multirow[t]{3}{*}{ Sign } & \multicolumn{4}{|c|}{$\begin{array}{l}\text { Dressing in the third } \\
\text { week }\end{array}$} & \multirow[t]{3}{*}{$\overline{\text { Sign }}$} \\
\hline & & & \multicolumn{2}{|c|}{$\begin{array}{c}\text { Pre- } \\
\text { breathing } \\
\text { exercises }\end{array}$} & \multicolumn{2}{|c|}{$\begin{array}{c}\text { Post- } \\
\text { breathing } \\
\text { exercises }\end{array}$} & & \multicolumn{2}{|c|}{$\begin{array}{c}\text { Pre- } \\
\text { breathing } \\
\text { exercises }\end{array}$} & \multicolumn{2}{|c|}{$\begin{array}{c}\text { Post- } \\
\text { breathing } \\
\text { exercises }\end{array}$} & \\
\hline & No & $\%$ & No & $\%$ & No & $\%$ & & No & $\%$ & No & $\%$ & \\
\hline Pain level first day & & & & & & & \multirow{6}{*}{$\begin{array}{l}\mathrm{F}=21.815 \\
\mathrm{p}=0.000^{*} *\end{array}$} & & & & & \multirow{6}{*}{$\begin{array}{l}\mathrm{F}=12.272 \\
\mathrm{p}=0.000 * *\end{array}$} \\
\hline No pain & 0 & 0.0 & 0 & 0.0 & 0 & 0.0 & & 0 & 0.0 & 0 & 0.0 & \\
\hline Mild & 0 & 0.0 & 0 & 0.0 & 0 & 0.0 & & 3 & 4.1 & 7 & 9.6 & \\
\hline Moderate & 3 & 4.1 & 16 & 21.9 & 39 & 53.4 & & 7 & 9.6 & 14 & 19.2 & \\
\hline Severe & 18 & 24.7 & 32 & 43.8 & 26 & 35.6 & & 34 & 46.6 & 35 & 47.9 & \\
\hline Worse & 52 & 71.2 & 25 & 34.2 & 8 & 11.0 & & 29 & 39.7 & 17 & 23.3 & \\
\hline Pain level second day & & & & & & & \multirow{6}{*}{$\begin{array}{c}\mathrm{F}=30.287 \\
\mathrm{p}=0.000 * *\end{array}$} & & & & & \multirow{6}{*}{$\begin{array}{l}\mathrm{F}=27.647 \\
\mathrm{p}=0.000 * *\end{array}$} \\
\hline No pain & 0 & 0.0 & 0 & 0.0 & 0 & 0.0 & & 0 & 0.0 & 0 & 0.0 & \\
\hline Mild & 0 & 0.0 & 0 & 0.0 & 0 & 0.0 & & 3 & 4.1 & 14 & 19.2 & \\
\hline Moderate & 2 & 2.7 & 7 & 9.6 & 25 & 34.2 & & 14 & 19.2 & 20 & 27.4 & \\
\hline Severe & 20 & 27.4 & 25 & 34.2 & 35 & 47.9 & & 34 & 46.6 & 36 & 49.3 & \\
\hline Worse & 51 & 69.9 & 41 & 56.2 & 13 & 17.8 & & 22 & 30.1 & 3 & 4.1 & \\
\hline Pain level third day & & & & & & & \multirow{6}{*}{$\begin{array}{c}\mathrm{F}=18.522 \\
\mathrm{p}=0.000^{*} *\end{array}$} & & & & & \multirow{6}{*}{$\begin{array}{c}\mathrm{F}=13.761 \\
\mathrm{p}=0.000 * *\end{array}$} \\
\hline No pain & 0 & 0.0 & 0 & 0.0 & 0 & 0.0 & & 1 & 1.4 & 1 & 1.4 & \\
\hline Mild & 0 & 0.0 & 0 & 0.0 & 0 & 0.0 & & 0 & 0.0 & 4 & 5.5 & \\
\hline Moderate & 0 & 0.0 & 3 & 4.1 & 25 & 34.2 & & 13 & 17.8 & 19 & 26.0 & \\
\hline Severe & 23 & 31.5 & 34 & 46.6 & 27 & 37.0 & & 32 & 43.8 & 32 & 43.8 & \\
\hline Worse & 50 & 68.5 & 36 & 49.3 & 21 & 28.8 & & 27 & 37.0 & 17 & 23.3 & \\
\hline Pain level fourth day & & & & & & & \multirow{6}{*}{$\begin{array}{l}\mathrm{F}=12.888 \\
\mathrm{p}=0.000 * *\end{array}$} & & & & & \multirow{6}{*}{$\begin{array}{c}\mathrm{F}=15.082 \\
\mathrm{p}=0.000 * *\end{array}$} \\
\hline No pain & 0 & 0.0 & 0 & 0.0 & 0 & 0.0 & & 1 & 1.4 & 1 & 1.4 & \\
\hline Mild & 0 & 0.0 & 0 & 0.0 & 2 & 2.7 & & 1 & 1.4 & 4 & 5.5 & \\
\hline Moderate & 0 & 0.0 & 5 & 6.8 & 18 & 24.7 & & 10 & 13.7 & 20 & 27.4 & \\
\hline Severe & 25 & 34.2 & 32 & 43.8 & 32 & 43.8 & & 31 & 42.5 & 36 & 49.3 & \\
\hline Worse & 48 & 65.8 & 36 & 49.3 & 21 & 28.8 & & 30 & 41.1 & 12 & 16.4 & \\
\hline
\end{tabular}

$P$-value $\leq 0.05$ 
Table (4): Continue.... UAB Pain Behavior Scale among the studied burned patients $(n=73)$

\begin{tabular}{|c|c|c|c|c|c|c|c|c|c|c|c|c|}
\hline \multirow[t]{3}{*}{ Variables } & \multirow{2}{*}{\multicolumn{2}{|c|}{$\begin{array}{c}\begin{array}{c}\text { Dressing in } \\
\text { First week }\end{array} \\
\text { Pain } \\
\text { assessment }\end{array}$}} & \multicolumn{4}{|c|}{ Dressing in the second week } & \multirow[t]{3}{*}{ Sign } & \multicolumn{4}{|c|}{$\begin{array}{l}\text { Dressing in the third } \\
\text { week }\end{array}$} & \multirow[t]{3}{*}{ Sign } \\
\hline & & & \multicolumn{2}{|c|}{$\begin{array}{l}\text { Pre- breathing } \\
\text { exercises }\end{array}$} & \multicolumn{2}{|c|}{$\begin{array}{c}\text { Post- breathing } \\
\text { exercises }\end{array}$} & & \multicolumn{2}{|c|}{$\begin{array}{c}\text { Pre- } \\
\text { breathing } \\
\text { exercises }\end{array}$} & \multicolumn{2}{|c|}{$\begin{array}{c}\text { Post- } \\
\text { breathing } \\
\text { exercises }\end{array}$} & \\
\hline & No & $\%$ & No & $\%$ & No & $\%$ & & No & $\%$ & No & $\%$ & \\
\hline 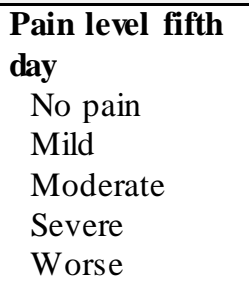 & $\begin{array}{c}0 \\
0 \\
7 \\
17 \\
49\end{array}$ & $\begin{array}{c}0.0 \\
0.0 \\
9.6 \\
23.3 \\
67.1\end{array}$ & $\begin{array}{c}0 \\
2 \\
8 \\
32 \\
31\end{array}$ & $\begin{array}{c}0.0 \\
2.7 \\
11.0 \\
43.8 \\
42.5\end{array}$ & $\begin{array}{c}0 \\
2 \\
17 \\
36 \\
18\end{array}$ & $\begin{array}{c}0.0 \\
2.7 \\
23.3 \\
49.3 \\
24.7\end{array}$ & $\begin{array}{c}\mathrm{F}=18.314 \\
\mathrm{p}=0.000 * *\end{array}$ & $\begin{array}{c}1 \\
3 \\
18 \\
26 \\
25\end{array}$ & $\begin{array}{c}1.4 \\
4.1 \\
24.7 \\
35.6 \\
34.2\end{array}$ & $\begin{array}{c}2 \\
7 \\
22 \\
37 \\
5\end{array}$ & $\begin{array}{c}2.7 \\
9.6 \\
30.1 \\
50.7 \\
6.8\end{array}$ & $\begin{array}{c}\mathrm{F}=21.711 \\
\mathrm{p}=0.000 * *\end{array}$ \\
\hline $\begin{array}{l}\text { Pain level six } \\
\text { day } \\
\text { No pain } \\
\text { Mild } \\
\text { Moderate } \\
\text { Severe } \\
\text { Worse }\end{array}$ & $\begin{array}{c}0 \\
0 \\
3 \\
25 \\
45\end{array}$ & $\begin{array}{c}0.0 \\
0.0 \\
4.1 \\
34.2 \\
61.6\end{array}$ & $\begin{array}{c}0 \\
2 \\
5 \\
36 \\
30\end{array}$ & $\begin{array}{c}0.0 \\
2.7 \\
6.8 \\
49.3 \\
41.1\end{array}$ & $\begin{array}{c}0 \\
4 \\
18 \\
34 \\
17\end{array}$ & $\begin{array}{c}0.0 \\
5.5 \\
24.7 \\
46.6 \\
23.3\end{array}$ & $\begin{array}{c}\mathrm{F}=8.568 \\
\mathrm{p}=0.000 * *\end{array}$ & $\begin{array}{c}1 \\
1 \\
8 \\
33 \\
30\end{array}$ & $\begin{array}{c}1.4 \\
1.4 \\
11.0 \\
45.2 \\
41.1\end{array}$ & $\begin{array}{c}1 \\
7 \\
22 \\
35 \\
8\end{array}$ & $\begin{array}{c}1.4 \\
9.6 \\
30.1 \\
47.9 \\
11.0\end{array}$ & $\begin{array}{c}\mathrm{F}=16.889 \\
\mathrm{p}=0.000 * *\end{array}$ \\
\hline $\begin{array}{l}\text { Pain level seven } \\
\text { day } \\
\text { No pain } \\
\text { Mild } \\
\text { Moderate } \\
\text { Severe } \\
\text { Worse }\end{array}$ & $\begin{array}{c}0 \\
0 \\
2 \\
26 \\
45\end{array}$ & $\begin{array}{c}0 \\
0 \\
2.7 \\
35.6 \\
61.6\end{array}$ & $\begin{array}{c}0 \\
1 \\
7 \\
33 \\
32\end{array}$ & $\begin{array}{c}0.0 \\
1.4 \\
9.6 \\
45.2 \\
43.8\end{array}$ & $\begin{array}{c}0 \\
6 \\
16 \\
32 \\
19\end{array}$ & $\begin{array}{c}0.0 \\
8.2 \\
21.9 \\
43.8 \\
26.0\end{array}$ & $\begin{array}{c}\mathrm{F}=20.149 \\
\mathrm{p}=0.000 * *\end{array}$ & $\begin{array}{c}1 \\
3 \\
19 \\
29 \\
21\end{array}$ & $\begin{array}{c}1.4 \\
4.1 \\
26.0 \\
39.7 \\
28.8\end{array}$ & $\begin{array}{c}2 \\
8 \\
32 \\
26 \\
5\end{array}$ & $\begin{array}{c}2.7 \\
11.0 \\
43.8 \\
35.6 \\
6.8\end{array}$ & $\begin{array}{c}\mathrm{F}=19.457 \\
\mathrm{p}=0.000 * *\end{array}$ \\
\hline
\end{tabular}

Table (4): Continue.... UAB Pain Behavior Scale among the studied burned patients $(n=73)$

\begin{tabular}{|c|c|c|c|c|c|c|c|c|c|c|c|c|}
\hline \multirow{3}{*}{ Items } & \multicolumn{10}{|c|}{ Level of pain } & \multirow[t]{3}{*}{$\overline{\mathrm{X}^{2}}$} & \multirow[t]{3}{*}{$\overline{\text { p-value }}$} \\
\hline & \multicolumn{2}{|c|}{ No pain } & \multicolumn{2}{|c|}{ Mild } & \multicolumn{2}{|c|}{ Moderate } & \multicolumn{2}{|c|}{ Severe } & \multicolumn{2}{|c|}{ Worse } & & \\
\hline & No & $\%$ & No & $\%$ & No & $\%$ & No & $\%$ & No & $\%$ & & \\
\hline $\begin{array}{l}\text { First week } \\
\text { Second week (pre-breathing } \\
\text { exercise) } \\
\text { Third week (pre-breathing } \\
\text { exercise) }\end{array}$ & $\begin{array}{l}0 \\
0 \\
1\end{array}$ & $\begin{array}{l}0.0 \\
0.0 \\
1.4\end{array}$ & $\begin{array}{l}0 \\
0 \\
2\end{array}$ & $\begin{array}{l}0.0 \\
0.0 \\
2.8\end{array}$ & $\begin{array}{c}2 \\
5 \\
21\end{array}$ & $\begin{array}{c}2.8 \\
6.8 \\
28.8\end{array}$ & $\begin{array}{l}23 \\
45 \\
24\end{array}$ & $\begin{array}{l}31.5 \\
61.6 \\
32.9\end{array}$ & $\begin{array}{l}48 \\
23 \\
25\end{array}$ & $\begin{array}{l}65.8 \\
31.5 \\
34.2\end{array}$ & 50.485 & $0.000 * *$ \\
\hline $\begin{array}{l}\text { Second week (post-breathing } \\
\text { exercise) } \\
\text { Third week (post-breathing } \\
\text { exercise) }\end{array}$ & $\begin{array}{l}0 \\
1\end{array}$ & $\begin{array}{l}0.0 \\
1.4\end{array}$ & $\begin{array}{l}2 \\
7\end{array}$ & $\begin{array}{l}2.8 \\
9.6\end{array}$ & $\begin{array}{l}25 \\
28\end{array}$ & $\begin{array}{l}34.2 \\
38.4\end{array}$ & $\begin{array}{l}40 \\
33\end{array}$ & $\begin{array}{l}54.8 \\
45.2\end{array}$ & $\begin{array}{l}6 \\
4\end{array}$ & $\begin{array}{l}8.2 \\
5.5\end{array}$ & 50.619 & $0.000 * *$ \\
\hline
\end{tabular}


Table (5): Relaxed behavior among the studied burned patients in the first weeks $(n=$ 73)

\begin{tabular}{|c|c|c|c|c|c|c|c|c|c|c|c|c|c|c|}
\hline \multirow[t]{2}{*}{ Variable } & \multicolumn{2}{|c|}{$1^{\text {st }}$ day } & \multicolumn{2}{|c|}{$2^{\text {nd }}$ day } & \multicolumn{2}{|c|}{$3^{\text {rd }}$ day } & \multicolumn{2}{|c|}{$4^{\text {th }}$ day } & \multicolumn{2}{|c|}{$5^{\text {th }}$ day } & \multicolumn{2}{|c|}{$6^{\text {th }}$ day } & \multicolumn{2}{|c|}{$7^{\text {th }}$ day } \\
\hline & NO. & $\%$ & NO. & $\%$ & NO. & $\%$ & NO. & $\%$ & NO. & $\%$ & NO. & $\%$ & NO. & $\%$ \\
\hline $\begin{array}{l}\text { Verbal sound } \\
\text { Relaxed } \\
\text { Un-relaxed }\end{array}$ & $\begin{array}{l}12 \\
61\end{array}$ & $\begin{array}{l}16.4 \\
83.6\end{array}$ & $\begin{array}{l}13 \\
60\end{array}$ & $\begin{array}{l}17.8 \\
82.2\end{array}$ & $\begin{array}{c}6 \\
67\end{array}$ & $\begin{array}{c}8.2 \\
91.8\end{array}$ & $\begin{array}{c}6 \\
67\end{array}$ & $\begin{array}{c}8.2 \\
91.8\end{array}$ & $\begin{array}{c}7 \\
66\end{array}$ & $\begin{array}{c}9.6 \\
90.4\end{array}$ & $\begin{array}{c}6 \\
67\end{array}$ & $\begin{array}{c}8.2 \\
91.8\end{array}$ & $\begin{array}{c}6 \\
67\end{array}$ & $\begin{array}{c}8.2 \\
91.8\end{array}$ \\
\hline $\begin{array}{l}\text { Body position } \\
\text { Relaxed } \\
\text { Un-relaxed }\end{array}$ & $\begin{array}{l}12 \\
61\end{array}$ & $\begin{array}{l}16.4 \\
83.6\end{array}$ & $\begin{array}{l}13 \\
60\end{array}$ & $\begin{array}{l}17.8 \\
82.2\end{array}$ & $\begin{array}{c}6 \\
67\end{array}$ & $\begin{array}{c}8.2 \\
91.8\end{array}$ & $\begin{array}{c}6 \\
67\end{array}$ & $\begin{array}{c}8.2 \\
91.8\end{array}$ & $\begin{array}{c}7 \\
66\end{array}$ & $\begin{array}{c}9.6 \\
90.4\end{array}$ & $\begin{array}{c}6 \\
67\end{array}$ & $\begin{array}{c}8.2 \\
91.8\end{array}$ & $\begin{array}{c}6 \\
67\end{array}$ & $\begin{array}{c}8.2 \\
91.8\end{array}$ \\
\hline $\begin{array}{l}\text { Head motion } \\
\text { Relaxed } \\
\text { Un-relaxed }\end{array}$ & $\begin{array}{l}12 \\
61\end{array}$ & $\begin{array}{l}16.4 \\
83.6\end{array}$ & $\begin{array}{l}13 \\
60\end{array}$ & $\begin{array}{l}17.8 \\
82.2\end{array}$ & $\begin{array}{c}6 \\
67\end{array}$ & $\begin{array}{c}8.2 \\
91.8\end{array}$ & $\begin{array}{c}6 \\
67 \\
\end{array}$ & $\begin{array}{c}8.2 \\
91.8\end{array}$ & $\begin{array}{c}7 \\
66\end{array}$ & $\begin{array}{c}9.6 \\
90.4\end{array}$ & $\begin{array}{c}6 \\
67\end{array}$ & $\begin{array}{c}8.2 \\
91.8\end{array}$ & $\begin{array}{c}6 \\
67\end{array}$ & $\begin{array}{c}8.2 \\
91.8\end{array}$ \\
\hline $\begin{array}{l}\text { Eyes } \\
\text { Relaxed } \\
\text { Un-relaxed }\end{array}$ & $\begin{array}{l}12 \\
61\end{array}$ & $\begin{array}{l}16.4 \\
83.6\end{array}$ & $\begin{array}{l}13 \\
60\end{array}$ & $\begin{array}{l}17.8 \\
82.2\end{array}$ & $\begin{array}{c}6 \\
67\end{array}$ & $\begin{array}{c}8.2 \\
91.8\end{array}$ & $\begin{array}{c}6 \\
67\end{array}$ & $\begin{array}{c}8.2 \\
91.8\end{array}$ & $\begin{array}{c}7 \\
66\end{array}$ & $\begin{array}{c}9.6 \\
90.4\end{array}$ & $\begin{array}{c}6 \\
67\end{array}$ & $\begin{array}{c}8.2 \\
91.8\end{array}$ & $\begin{array}{c}6 \\
67\end{array}$ & $\begin{array}{c}8.2 \\
91.8\end{array}$ \\
\hline $\begin{array}{l}\text { Mouth } \\
\text { Relaxed } \\
\text { Un-relaxed }\end{array}$ & $\begin{array}{l}12 \\
61\end{array}$ & $\begin{array}{l}16.4 \\
83.6\end{array}$ & $\begin{array}{l}13 \\
60\end{array}$ & $\begin{array}{l}17.8 \\
82.2\end{array}$ & $\begin{array}{c}6 \\
67\end{array}$ & $\begin{array}{c}8.2 \\
91.8\end{array}$ & $\begin{array}{c}6 \\
67\end{array}$ & $\begin{array}{c}8.2 \\
91.8\end{array}$ & $\begin{array}{c}7 \\
66\end{array}$ & $\begin{array}{c}9.6 \\
90.4\end{array}$ & $\begin{array}{c}6 \\
67\end{array}$ & $\begin{array}{c}8.2 \\
91.8\end{array}$ & $\begin{array}{c}6 \\
67\end{array}$ & $\begin{array}{c}8.2 \\
91.8\end{array}$ \\
\hline
\end{tabular}


Table (5): Continue....relaxed behavior among the studied burned patients in the second week $(\mathrm{n}=73)$

\begin{tabular}{|c|c|c|c|c|c|c|c|c|c|c|c|c|c|c|c|c|c|c|c|c|c|c|c|c|c|c|c|c|}
\hline \multirow[t]{3}{*}{ Variables } & \multicolumn{4}{|c|}{$1^{\text {st }}$ day } & \multicolumn{4}{|c|}{$2^{\text {nd }}$ day } & \multicolumn{4}{|c|}{$3^{\text {rd }}$ day } & \multicolumn{4}{|c|}{$4^{\text {th }}$ day } & \multicolumn{4}{|c|}{$5^{\text {th }}$ day } & \multicolumn{4}{|c|}{$6^{\text {th }}$ day } & \multicolumn{4}{|c|}{$7^{\text {th }}$ day } \\
\hline & \multicolumn{2}{|c|}{ Pre } & \multicolumn{2}{|c|}{ Post } & \multicolumn{2}{|c|}{ Pre } & \multicolumn{2}{|c|}{ Post } & \multicolumn{2}{|c|}{ Pre } & \multicolumn{2}{|c|}{ Post } & \multicolumn{2}{|c|}{ Pre } & \multicolumn{2}{|c|}{ Post } & \multicolumn{2}{|c|}{ Pre } & \multicolumn{2}{|c|}{ Post } & \multicolumn{2}{|c|}{ Pre } & \multicolumn{2}{|c|}{ Post } & \multicolumn{2}{|c|}{ Pre } & \multicolumn{2}{|c|}{ Post } \\
\hline & No & $\%$ & No & $\%$ & No & $\%$ & No & $\%$ & No & $\%$ & No & $\%$ & No & $\%$ & No & $\%$ & No & $\%$ & No & $\%$ & No & $\%$ & No & $\%$ & No & $\%$ & No & $\%$ \\
\hline $\begin{array}{l}\text { Verbal sound } \\
\text { Relaxed }\end{array}$ & 6 & 8.2 & 27 & 37.0 & 16 & 21.9 & 39 & 53.4 & 17 & 23.3 & 40 & 54.8 & 19 & 26.0 & 41 & 56.2 & 19 & 26.0 & 41 & 56.2 & 19 & 26.0 & 44 & 60.3 & 19 & 26.0 & 44 & 60.3 \\
\hline Un-relaxed & 67 & 91.8 & 46 & 63.0 & 57 & 78.1 & 34 & 46.6 & 56 & 76.7 & 33 & 45.2 & 54 & 74.0 & 32 & 43.8 & 54 & 74.0 & 32 & 43.8 & 54 & 74.0 & 29 & 39.7 & 54 & 74.0 & 29 & 39.7 \\
\hline Body position & & & & & & & & & & & & & & & & & & & & & & & & & & & & \\
\hline Relaxed & 6 & 8.2 & 27 & 37.0 & 16 & 21.9 & 39 & 53.4 & 17 & 23.3 & 40 & 54.8 & 19 & 26.0 & 41 & 56.2 & 19 & 26.0 & 41 & 56.2 & 19 & 26.0 & 44 & 60.3 & 19 & 26.0 & 44 & 60.3 \\
\hline Un-relaxed & 67 & 91.8 & 46 & 63.0 & 57 & 78.1 & 34 & 46.6 & 56 & 76.7 & 33 & 45.2 & 54 & 74.0 & 32 & 43.8 & 54 & 74.0 & 32 & 43.8 & 54 & 74.0 & 29 & 39.7 & 54 & 74.0 & 29 & 39.7 \\
\hline Head motion & & & & & & & & & & & & & & & & & & & & & & & & & & & & \\
\hline Relaxed & 6 & 8.2 & 27 & 37.0 & 16 & 21.9 & 39 & 53.4 & 17 & 23.3 & 40 & 54.8 & 19 & 26.0 & 41 & 56.2 & 19 & 26.0 & 41 & 56.2 & 19 & 26.0 & 44 & 60.3 & 19 & 26.0 & 44 & 60.3 \\
\hline Un-relaxed & 67 & 91.8 & 46 & 63.0 & 57 & 78.1 & 34 & 46.6 & 56 & 76.7 & 33 & 45.2 & 54 & 74.0 & 32 & 43.8 & 54 & 74.0 & 32 & 43.8 & 54 & 74.0 & 29 & 39.7 & 54 & 74.0 & 29 & 39.7 \\
\hline Eyes & & & & & & & & & & & & & & & & & & & & & & & & & & & & \\
\hline Relaxed & 6 & 8.2 & 27 & 37.0 & 16 & 21.9 & 39 & 53.4 & 17 & 23.3 & 40 & 54.8 & 19 & 26.0 & 41 & 56.2 & 19 & 26.0 & 41 & 56.2 & 19 & 26.0 & 44 & 60.3 & 19 & 26.0 & 44 & 60.3 \\
\hline Un-relaxed & 67 & 91.8 & 46 & 63.0 & 57 & 78.1 & 34 & 46.6 & 56 & 76.7 & 33 & 45.2 & 54 & 74.0 & 32 & 43.8 & 54 & 74.0 & 32 & 43.8 & 54 & 74.0 & 29 & 39.7 & 54 & 74.0 & 29 & 39.7 \\
\hline Mouth & & & & & & & & & & & & & & & & & & & & & & & & & & & & \\
\hline Relaxed & 6 & 8.2 & 27 & 37.0 & 16 & 21.9 & 39 & 53.4 & 17 & 23.3 & 40 & 54.8 & 19 & 26.0 & 41 & 56.2 & 19 & 26.0 & 41 & 56.2 & 19 & 26.0 & 44 & 60.3 & 19 & 26.0 & 44 & 60.3 \\
\hline Un-relaxed & 67 & 91.8 & 46 & 63.0 & 57 & 78.1 & 34 & 46.6 & 56 & 76.7 & 33 & 45.2 & 54 & 74.0 & 32 & 43.8 & 54 & 74.0 & 32 & 43.8 & 54 & 74.0 & 29 & 39.7 & 54 & 74.0 & 29 & 39.7 \\
\hline
\end{tabular}


Table (5): Continue.... relaxed behavior among the studied burned patients in the third week $(\mathrm{n}=73)$

\begin{tabular}{|c|c|c|c|c|c|c|c|c|c|c|c|c|c|c|c|c|c|c|c|c|c|c|c|c|c|c|c|c|}
\hline \multirow[t]{3}{*}{ Variables } & \multicolumn{4}{|c|}{$1^{\text {st }}$ day } & \multicolumn{4}{|c|}{$2^{\text {nd }}$ day } & \multicolumn{4}{|c|}{$3^{\text {rd }}$ day } & \multicolumn{4}{|c|}{$4^{\text {th }}$ day } & \multicolumn{4}{|c|}{$5^{\text {th }}$ day } & \multicolumn{4}{|c|}{$\overline{6}^{\text {th }}$ day } & \multicolumn{4}{|c|}{$7^{\text {th }}$ day } \\
\hline & \multicolumn{2}{|c|}{ Pre } & \multicolumn{2}{|c|}{ Post } & \multicolumn{2}{|c|}{ Pre } & \multicolumn{2}{|c|}{ Post } & \multicolumn{2}{|c|}{ Pre } & \multicolumn{2}{|c|}{ Post } & \multicolumn{2}{|c|}{ Pre } & \multicolumn{2}{|c|}{ Post } & \multicolumn{2}{|c|}{\begin{tabular}{l|l} 
Pre & \\
\end{tabular}} & \multicolumn{2}{|c|}{ Post } & \multicolumn{2}{|c|}{\begin{tabular}{l|l} 
Pre & \\
\end{tabular}} & \multicolumn{2}{|c|}{ Post } & \multicolumn{2}{|c|}{\begin{tabular}{l|l} 
Pre \\
\end{tabular}} & \multicolumn{2}{|c|}{ Post } \\
\hline & No & $\%$ & No & $\%$ & No & $\%$ & No & $\%$ & No & $\%$ & No & $\%$ & No & $\%$ & No & $\%$ & No & $\%$ & No & $\%$ & No & $\%$ & No & $\%$ & No & $\%$ & No & $\%$ \\
\hline & & & 4 & & 21 & & 46 & 3.0 & 24 & 2.9 & 8 & & 25 & 4.2 & 50 & & 9 & & 61 & & 40 & & 62 & 4.9 & 40 & & 62 & \\
\hline Un-re & & 4.0 & 29 & & 2 & 71.2 & 27 & 7.0 & 49 & 67.1 & 5 & 34.2 & 48 & 5.8 & 23 & & 34 & 46.6 & 12 & 16.4 & 33 & 45.2 & 11 & 15.1 & 33 & 45.2 & 11 & 15. \\
\hline & & 26.0 & 44 & 60.3 & 21 & 28.8 & 46 & 63.0 & 24 & 32.9 & 48 & 65.8 & 25 & 34.2 & 50 & 68.5 & 39 & |53.4 & 61 & 83.6 & 40 & 54.8 & 62 & 84.9 & 40 & 54.8 & 62 & 84.9 \\
\hline Un-rela & & 74.0 & 29 & 9.7 & 52 & 71.2 & 27 & 37.0 & 49 & 67.1 & 25 & 34.2 & 48 & 65.8 & 23 & 31.5 & 34 & 46.6 & 12 & 16.4 & 33 & 45.2 & 11 & 15.1 & 33 & 45.2 & 11 & 15.1 \\
\hline & & 26.0 & 44 & 60.3 & 21 & 28.8 & 46 & 63.0 & 24 & 32.9 & 48 & 65.8 & 25 & 34.2 & 50 & 68.5 & 39 & 53.4 & 61 & 83.6 & 40 & 54.8 & 62 & 84.9 & 40 & 54.8 & 62 & 84.9 \\
\hline Un-re & & 74.0 & 29 & 39.7 & 52 & 71.2 & 27 & 37.0 & 49 & 67.1 & 25 & 34.2 & 48 & 65.8 & 23 & 31.5 & 34 & 46.6 & 12 & 16.4 & 33 & 45.2 & 11 & 15.1 & 33 & 45.2 & 11 & 15.1 \\
\hline & & 6.0 & 44 & & 21 & 28.8 & 46 & 63.0 & 24 & 32.9 & 48 & & 25 & 34.2 & 50 & & 39 & 53.4 & 61 & 3.6 & 40 & 54.8 & 62 & 84.9 & 40 & 54.8 & 62 & 04. \\
\hline & & 74.0 & 29 & 39.7 & 52 & 71.2 & 27 & 37.0 & 49 & 67.1 & 25 & 34.2 & 48 & 65.8 & 23 & 31.5 & 34 & 46.6 & 12 & 16.4 & 33 & 45.2 & 11 & 15.1 & 33 & 45.2 & 11 & 15. \\
\hline & & & & & & & & & & & & & & & & & & & & & & & & & & & & \\
\hline & & 26.0 & 44 & 60.3 & 21 & 28.8 & 46 & 63.0 & 24 & 32.9 & 48 & 65.8 & 25 & 34.2 & 50 & 68.5 & 39 & 53.4 & 61 & 83.6 & 40 & 54.8 & 62 & 84.9 & 40 & 54.8 & 62 & 84.9 \\
\hline Un-relaxed & 54 & 74.0 & 29 & 39.7 & 52 & 71.2 & 27 & 37.0 & 49 & 67.1 & 25 & 34.2 & 48 & 65.8 & 23 & 31.5 & 34 & 46.6 & 12 & 16.4 & 33 & 45.2 & 11 & 15.1 & 33 & 45.2 & 11 & 15.1 \\
\hline
\end{tabular}

Table (5): Continue....total score of the burned patients regarding to their relaxed behavior first, second and third week $(\mathrm{n}=73)$.

\begin{tabular}{|c|c|c|c|c|c|c|}
\hline \multirow{3}{*}{ Items } & \multicolumn{4}{|c|}{ Total relaxed behavior } & \multirow[t]{3}{*}{$\mathbf{X}^{2}$} & \multirow[t]{3}{*}{ p-value } \\
\hline & \multicolumn{2}{|c|}{ Relax } & \multicolumn{2}{|c|}{ Un-relax } & & \\
\hline & No & $\%$ & No & $\%$ & & \\
\hline First week & 3 & 4.1 & 70 & 95.9 & \multirow{3}{*}{14.787} & \multirow{3}{*}{$0.001 *$} \\
\hline Second week (pre-breathing exercises) & 16 & 21.9 & 57 & 78.1 & & \\
\hline Third week (pre-breathing exercises) & 20 & 27.4 & 53 & 72.6 & & \\
\hline Second week (post- use of bre athing exercises) & 38 & 52.1 & 35 & 47.9 & \multirow{2}{*}{12.311} & \multirow{2}{*}{$0.002 *$} \\
\hline Third week (post-bre athing exercises) & 46 & 63.0 & 27 & 37.0 & & \\
\hline
\end{tabular}


Table (6): Vital signs among the studied burned patients in first weeks ( $n=73$ )

\begin{tabular}{|c|c|c|c|c|c|c|c|}
\hline Variable & $\overline{1^{\text {st }} \text { day }}$ & $2^{\text {nd }}$ day & $3^{\text {rd day }}$ & 4 $^{\text {th }}$ day & $\overline{5^{\text {th }} \text { day }}$ & 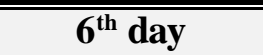 & $\overline{7^{\text {th }}}$ day \\
\hline $\mathbf{B P}($ systolic $)$ & $152.64 \pm 18.17$ & $147.85 \pm 20.14$ & $147.42 \pm 17.14$ & $152.11 \pm 15.64$ & $147.29 \pm 17.98$ & $151.60 \pm 16.97$ & $147.36 \pm 16.55$ \\
\hline BP (diastolic) & $82.54 \pm 8.88$ & $84.39 \pm 8.50$ & $82.88 \pm 8.53$ & $84.81 \pm 8.96$ & $84.10 \pm 7.80$ & $83.26 \pm 7.23$ & $84.10 \pm 6.78$ \\
\hline Total dias tolic & $83.72 \pm 4.94$ & $\mathrm{t}=3.019$ & \multicolumn{5}{|c|}{$\mathrm{p}=0.004^{*}$} \\
\hline Pulse rate & $94.49 \pm 10.2$ & $94.49 \pm 10.2$ & $94.49 \pm 10.24$ & $94.49 \pm 10.24$ & $94.49 \pm 10.24$ & $94.49 \pm 10.24$ & $94.49 \pm 10.24$ \\
\hline Total pulse rate & \multicolumn{2}{|c|}{$94.49 \pm 10.24$} & $\mathrm{t}=5.837$ & \multicolumn{4}{|c|}{$\mathrm{p}=0.000^{* *}$} \\
\hline Tempe rature & $36.62 \pm 0.42$ & $36.62 \pm 0.42$ & $36.62 \pm 0.42$ & $36.62 \pm 0.42$ & $36.62 \pm 0.47$ & $36.62 \pm 0.42$ & $36.62 \pm 0.42$ \\
\hline Total temp & \multicolumn{2}{|c|}{$36.63 \pm 0.41$} & $\mathrm{t}=0.376$ & \multicolumn{4}{|c|}{$\mathrm{p}=0.71$} \\
\hline
\end{tabular}


Table (6): Continue.... vital signs among the studied burned patients in second weeks $(n=73)$ before and after use of breathing exercises $(n=73)$

\begin{tabular}{|c|c|c|c|c|c|c|c|c|c|c|c|c|c|c|}
\hline \multirow[t]{3}{*}{ "Variables } & \multicolumn{2}{|c|}{ 1 $^{\text {st }}$ day } & \multicolumn{2}{|c|}{$2^{\text {nd }}$ day } & \multicolumn{2}{|c|}{$\overline{3}^{\text {rd day }}$} & \multicolumn{2}{|c|}{$\overline{4^{\text {th }} \text { day }}$} & \multicolumn{2}{|c|}{$\overline{5}^{\text {th }}$ day } & \multicolumn{2}{|c|}{$\overline{\text { 6 }^{\text {th }} \text { day }}$} & \multicolumn{2}{|c|}{$\overline{7}^{\text {th }}$ day } \\
\hline & Pre & Post & Pre & Post & Pre & Post & Pre & Post & Pre & Post & Pre & Post & Pre & Post \\
\hline & $\begin{array}{c}\text { Mean } \pm \\
\text { SD }\end{array}$ & $\begin{array}{c}\text { Mean } \pm \\
\text { SD }\end{array}$ & $\begin{array}{c}\text { Mean } \pm \\
\text { SD }\end{array}$ & $\begin{array}{c}\text { Mean } \pm \\
\text { SD }\end{array}$ & Mean \pm SD & Mean \pm SD & Mean \pm SD & Mean \pm SD & Mean \pm SD & Mean \pm SD & Mean \pm SD & Mean \pm SD & Mean \pm SD & $\begin{array}{c}\text { Mean } \pm \\
\text { SD }\end{array}$ \\
\hline BP (systolic) & $153.54 \pm 16.04$ & \begin{tabular}{|l|}
$146.78 \pm 16.09$ \\
\end{tabular} & $151.94 \pm 15.46$ & $147.29 \pm 15.45$ & $153.61 \pm 16.04$ & $146.58 \pm 15.89$ & $153.61 \pm 16.04$ & $146.58 \pm 15.90$ & $153.61 \pm 16.04$ & $146.58 \pm 15.9$ & $151.94 \pm 15.46$ & $147.29 \pm 15.45$ & \begin{tabular}{|l|}
$153.61 \pm 16.04$ \\
\end{tabular} & $\begin{array}{c}146.58 \pm 15.9 \\
0\end{array}$ \\
\hline Total systolic & \multicolumn{2}{|c|}{$153.13 \pm 11.80$} & \multicolumn{2}{|c|}{$146.81 \pm 11.64$} & $t=5.961$ & $\mathrm{p}=0.000^{* *}$ & & & & & & & & \\
\hline BP (diastolic) & $83.19 \pm 7.47$ & $79.03 \pm 6.90$ & $83.75 \pm 6.91$ & $81.88 \pm 6.58$ & $83.03 \pm 7.89$ & $79.03 \pm 6.90$ & $83.03 \pm 7.89$ & $79.03 \pm 6.90$ & $83.03 \pm 7.89$ & $79.03 \pm 6.90$ & $83.75 \pm 6.91$ & $81.88 \pm 6.58$ & $83.03 \pm 7.89$ & $79.03 \pm 6.90$ \\
\hline Total diastolic & \multicolumn{2}{|c|}{$83.26 \pm 5.75$} & \multicolumn{2}{|c|}{$79.84 \pm 5.49$} & $\mathrm{t}=6.411$ & $\mathrm{p}=0.000^{* *}$ & & & & & & & & \\
\hline $\begin{array}{c}\text { Respiration } \\
\text { rate }\end{array}$ & $26.03 \pm 1.83$ & $24.75 \pm 1.18$ & $25.73 \pm 1.98$ & $24.63 \pm 1.31$ & $25.58 \pm 1.90$ & $24.60 \pm 1.34$ & $25.58 \pm 1.90$ & $24.60 \pm 1.34$ & $25.58 \pm 1.90$ & $24.60 \pm 1.34$ & $25.58 \pm 1.90$ & $24.60 \pm 1.34$ & $25.58 \pm 1.90$ & $24.60 \pm 1.34$ \\
\hline $\begin{array}{c}\text { Total } \\
\text { respiration }\end{array}$ & \multicolumn{2}{|c|}{$25.66 \pm 1.81$} & \multicolumn{2}{|c|}{$24.63 \pm 1.30$} & $t=7.371$ & $\mathrm{p}=0.000^{* *}$ & & & & & & & & \\
\hline Pulse rate & $94.49 \pm 10.2$ & $90.40 \pm 8.26$ & $94.49 \pm 10.2$ & $90.40 \pm 8.26$ & $94.49 \pm 10.24$ & $90.40 \pm 8.26$ & $94.49 \pm 10.24$ & $90.40 \pm 8.26$ & $94.49 \pm 10.24$ & $90.40 \pm 8.26$ & $94.49 \pm 10.24$ & $90.40 \pm 8.26$ & $94.49 \pm 10.24$ & $90.40 \pm 8.26$ \\
\hline $\begin{array}{c}\text { Total pulse } \\
\text { rate }\end{array}$ & $94.49 \pm 10.2$ & $90.40 \pm 8.26$ & $t=5.837$ & $\mathrm{p}=0.000^{* *}$ & & & & & & & & & & \\
\hline Temperature & $36.66 \pm 0.47$ & $36.62 \pm 0.42$ & $36.62 \pm 0.42$ & $36.62 \pm 0.42$ & $36.62 \pm 0.42$ & $36.62 \pm 0.42$ & $36.62 \pm 0.42$ & $36.62 \pm 0.42$ & $36.62 \pm 0.42$ & $36.62 \pm 0.42$ & $36.62 \pm 0.46$ & $36.62 \pm 0.49$ & $36.62 \pm 0.42$ & $36.62 \pm 0.42$ \\
\hline Total temp & \multicolumn{2}{|c|}{$36.63 \pm 0.41$} & \multicolumn{2}{|c|}{$36.62 \pm 0.42$} & $t=1.000$ & $\mathrm{p}=0.32$ & & & & & & & & \\
\hline
\end{tabular}




\section{DISCUSSION:}

Burn is a main reason for acute damages and constitutes more than one percent of burden of diseases. Burn damages are important causes of mortality and morbidity. They can also lead to many physical, psychological, social, and economic problems. The prevalence of burn is high, so that its associated mortality and permanent morbidity are reported frequently. Also, the treatment of burn injuries is a long-term process that requires repeated surgical interventions and ongoing medical treatments by taking a lot of time and imposing heavy cost to community (Brigham \& Dimick, 2008). According to the high prevalence of burn damages, it is necessary to evaluate different treatment methods in order to reduce pain and control its physical and psychological complications

(Bruckenthal, 2010).

The treatment of burn patients is very challenging because burn injuries are one of the most severe traumas that can be experienced. As medical technology has advanced, the majority of burn patients are now being successfully resuscitated and typically undergo early escharotomy, skin transplantation, and antibiotic administration in addition to receiving nutritional support, which together dramatically decrease their mortality rate (Burke, Mohn-Brown \& Eby, 2011).

Burn wounds can be particularly painful and stressful for patients, particularly during dressing change and other aspects of wound care. Research into other types of wounds has demonstrated that stress and pain at dressing change are closely linked and related to healing, since high levels of stress and pain are associated with a longer length of time for a wound to heal (Culleiton \& Simko, 2013).

Breathing exercise is a simple behavioral intervention to manage pain and anxiety. However, the information about the effects of relaxation breathing on pain and anxiety levels for burn patients during dressing changes is limited (Burke, et al. 2011).

The result of the current study revealed that, the mean age of patients was $(28.79 \pm 8.95)$ years, and the highest of them aged from 20 to less than 30years old. On the other hand Joo, Lee, Cho, Lee, and Seo, (2020) found that, the larger part of subjects were in age between 18-40 years.

In connection to sex, $66 \%$ of the contemplated test was male. This outcome was negated with (Gibson, Gallagher, \& Heffernan, 2019) expressed that, ladies were for the most part at higher hazard for consume than men, particularly in more youthful age gatherings. 
As respects to conjugal status, the most astounding rate was married, this result was in harmonious with (Elsherbiny, Salem, El-Sabbagh, Elhadidy, \& Eldeen, 2011); (Joo et al., 2020), who found that, the lion's share of consumed patients were hitched. Then again, Edwards, (2013) detailed that, the greater part of single grown-up most normally influenced with consume damage.

Concerning level of training, the most elevated level of them had fundamental instruction. From the perspective of the scientist, this gathering had absence of information in regards to security precautionary measures that expansion danger of consume damage. This finding compatible with (Joo et al., 2020), who demonstrated that, around half of consumed patients had essential and auxiliary instruction. Then again, (Elsherbiny et al., 2011) expressed that, the dominant part of consumed subjects were ignorant; this outcome was conversely with the consequence of present investigation.

In connection to living arrangement, the most noteworthy rate was from country territories. From the perspective of the scientist, this can be legitimized by absence of security safeguard application in country territory which can open individuals to higher danger of consume wounds additionally deficient wellbeing administrations in provincial region, moreover, unsupervised and imprudent treatment of gas funnels without security highlights and lamp oil put away at the home that expansion danger of flame.

The present examination discoveries are in a similar line with (Morsy \& Alam, 2014), who saw that, the mean of age was $(33.40 \pm 12.68)$ years, the most elevated rate was guys, hitched, lived in rustic territory, had fundamental instruction, and the greater part of study subjects filling in as manual specialist.

In the current study, it was observed that there were statistically significant difference in relation to burn specific anxiety level throughout the intervention program phases before and after using breathing exercise during exchange dressing, this may be related to shifting breathing rate and pattern can stimulate the body's parasympathetic response. And this is the equally powerful and opposite system to the Emergency Response and is often called the relaxation response.

This result are in agreement with (Park, et al., 2013) who concluded that relaxation breathing is a simple and inexpensive technique nurses can use to help burn patients manage pain and anxiety during dressing changes. 
As yielded by the present study findings, there was a high statistically difference in pain level pre and post measurement; it clarifies that, breathing technique could decrease pain. This may be related to the patient focus his attention on his breath. He just notices the sensation of air going into and out of his lungs. This is the practice of mindfulness. The challenge with this practice is to maintain attention on his breath while thoughts, feelings and sensations arise. The trick is to just notice these and return to his breath.

These discoveries are bolstered by (Morsy, \& Alam, 2014) who found a noteworthy measurable distinction between the level of torment before and in the wake of dressing trade of consumed wound. It could be reasoned that, rehearsing of unwinding procedures and diversion assume a critical part in torment control amid dressing with blend of pharmacological techniques.

The present examination discoveries was upheld by Fakhar, Frafiee, Rohangiz, and Mehrnoosh, (2011) who found a contrast between the level of agony score when intercession in exploratory gathering who were perform jaw unwinding strategies.

However, the present study findings are in agreement with Park, et al., (2013), who stated that, relaxation breathing, is a simple and inexpensive technique nurses can use to help burn patients manage pain and anxiety during dressing changes.

In addition, Allorto, Wall and Clarke, (2018) demonstrate that both, interventions: (muscle relaxation and mental imagery) could significantly reduce pain intensity in the patients with the second-degree burn wound. While, lack of use of non-pharmacological interventions can lead to increased pain or inadequate pain control.

Numerous studies have shown that pain during dressing change is a major issue for patients with both acute and chronic wounds. Despite this evidence, pain is often misunderstood by clinicians and can be poorly managed. Pain can result in patients abandoning dressing regimens and clinicians need to ensure that patients have their pain reduced as much as possible and do not have to face the trauma of repeated painful dressing changes (Edward, 2013).

In addition, Alaa Eldin, et al., (2015) found a significant difference between pre and post relaxation breathing technique during dressing of burn wound on pain behavior scale in relation to facial grimaces, standing posture, mobility and all item of scales. Also, he reported that it was a 
significant difference between the study and control group as regard to facial grimace and muscle tone and scales behavior.

As observed by the current study findings, there was an improvement in anxiety post relaxation technique. This result is supported by (Morsy, \& Alam, 2014), who said that, the honing of unwinding systems and diversion can assume an imperative part in controlling nervousness amid dressing among consumed patients.

Finally, this study revealed to the benefit of relaxation breathing technique for hospitalized burned patients during wound dressing. It found out that the majority of patients reported significant decrease in their pain intensity and anxiety level during dressing of burn wound.

There is Improvement in relaxed behavior throughout the intervention program may be related to the effect of breathing exercise on decreasing anxiety and pain level during exchange dressing of burn wound

The findings of the present study revealed a significant difference in heart, respiration rate , systolic outcomes and diastolic blood pressure among studied patients throughout the intervention program between pre and post test. It could be an evidence that there was significant difference between bloods supporting of use the relaxation breathing technique in reducing pain care at wound. This is inconsistent with (Alaa Eldin, et al., 2015) who found the same result. In contrast, when comparing between study and control group regarding the benefits of relaxation breathing technique, it had a positive effect on respiration, systolic and diastolic blood pressure before, during and after wound care on third assessment. This is inconsistent with a study done on effectiveness of music intervention by de Jong, Middelkoop, Faber, and Van Loey, (2007) who stated that it is only decreased heart rate and did not improve other physiologic measures as respiration, systolic and diastolic blood pressures of burn injuries after wound care.

In addition, Rafii, Mohammadi-Fakhar and Jamshidi Orak, (2014) found a significant difference between pre and post relaxation breathing technique during dressing of burn wound on pain behavior scale in relation to facial grimaces, scales behavior, restlessness. Also, he reported that it was a significant difference between the study and control group as regard to facial and muscle tone and items of scales behavior. 
Finally, this study revealed to the benefit of relaxation breathing technique for hospitalized burned patients during wound dressing. It found out that the majority of patients reported significant decrease in their pain intensity and anxiety level during dressing of burn wound post practicing relaxation breathing technique. Which in turn with reflecting pain behavior scale significantly decreased post practicing relaxation breathing technique during dressing of burned wound . In addition, a significant improvement post practicing relaxation breathing technique during dressing of burned wound was found in respiration, heart rate and blood pressure.

Breathing exercises can help maintain and increase lung capacity, making it easier to keep lungs healthy and get body the oxygen it needs. So, in the current study use of breathing exercise during exchange dressing of burn wound lead to significant improvement in pulse, respiration and blood pressure.

These results are supported by de Jong and Gamel, (2006) who stated that adult patients with burns experience pain during wound care despite pharmacological interventions. Additional interventions are needed to improve the effectiveness of pain management. Relaxation techniques can be considered, for example breathing exercises, music and distraction. A simple breathing relaxation technique is especially relevant because it involves no risk, is easy and quick to learn, equipment does not need to be purchased and it can be employed immediately by the often exhausted and ill patient.

\section{CONCLUSION}

In the light of the present study findings:

It was concluded that, using of breathing exercise is very important to reduce pain and anxiety associated with burn dressing change for hospitalized burned patients.

\section{RECOMMENDATIONS}

Based on the findings of the present study, the following recommendations are to be considered:

\section{For nurses:}

1. Training programs should be applied for nurses in the burned care units to improve their knowledge and practice regarding developmental supportive care for burned patients.

2. Workshops should be developed by the hospital administrative authority for nurses about developmental supportive care needed for burn dressing. 
3. A procedure manual for the developmental supportive care for preterm and low birth weight infants in NICU needs to be developed and standardized.

4. Staff education about relaxation techniques and methods of providing support should be provided to burn patients.

5. Replication of this study at different places in Egypt, and developing an educational program and conduct it on a larger sample size, different geographical areas.

\section{REFERENCES}

Alaa Eldin, S., Mohamed H., \& Ragab I. (2015). Effect of relaxation breathing technique among patients with moderate burn on their pain and anxiety at wound care. World Journal of Nursing Sciences, 1 (3), 110-123.

Allorto, N., Wall, S. \& Clarke, D., (2018). Quantifying capacity for burn care in South Africa. Burns Open, 2(4), 188-192.

Gibson, C., Gallagher, J., \& Heffernan, J. (2019). Development of the Pan African burn services directory. Burns, 45(8), 1918-1922.

Asgharipour, N., Shariati, M. \& Borhani, M. (2017). Assessment of guided imagery effect on reducing anxiety and pain associated with wound dressing changes in burn patients. Iranian Journal of Psychiatry and Behavioral Sciences, 11(3).

Brigham, P. \& Dimick, A. (2008). The evolution of burn care facilities in the United States. Journal of Burn Care \& Research, 29(1), 248-256.

Bruckenthal, P., (2010). Integrating Nonpharmacologic and alternative strategies into a comprehensive management approach for older adults with pain. Pain Management Nursing, 11(2), S23-S31.

Burke, K., Mohn-Brown, E., \& Eby, L. (2011). Medical-surgical nursing care. Upper Saddle River, N.J.: Pearson.

Culleiton, A., \& Simko, L. (2013). Caring for patients with burn injuries. Nursing, 43(8), 26-34. 
de Jong, A., \& Gamel, C. (2006). Use of a simple relaxation technique in burn care: literature review. Journal of Advanced Nursing, 54(6), 710-721.

de Jong, A., Middelkoop, E., Faber, A., \& Van Loey, N. (2007). Non-pharmacological nursing interventions for procedural pain relief in adults with burns: A systematic literature review. Burns, 33(7), 811-827.

Douglas, H. E., \& Wood, F. (2017). Burns dressings. Australian family physician, 46(3), 94-97.

Elsherbiny, O. E. E., Salem, M. A., El-Sabbagh, A. H., Elhadidy, M. R., \& Eldeen, S. M. A. (2011). Quality of life of adult patients with severe burns. Burns, 37(5), 776-789.

Edwards, J. (2013). Dealing with wound-related pain at dressing change. Journal of Community Nursing, 27(4), 36-42.

Fakhar, F., Frafiee, A., Rohangiz, J., \& Mehrnoosh, I. (2011). The effect of jaw relaxation on pain anxiety of burn dressing: Randomized clinical trial with control group $. I J N R, 5(19), 58-67$.

Hughes, O., MacQuhae, F., Rakosi, A., Herskovitz, I. \& Kirsner, R., (2016). Stress and wound healing. Stress and Skin Disorders,Pp. 185-207.

Joo, S., Lee, S., Cho, Y., Lee, K., \& Seo, C, (2020). Effects of robot-assisted gait training in patients with burn injury on lower extremity: A Single-Blind, randomized controlled trial. Journal of Clinical Medicine, 9(9), 2813.

Lynn, P. (2015). Taylor's Clinical Nursing Skills: A Nursing Process Approach (4th ed.). p.360

Morsy, N., \& Alam, Z.A. (2014). Effect of Relaxation Techniques on Pain Associated with Dressing among Moderately Burned Patients, unpublished Master thesis. Faculty of nursing, Tanta University. Available at https://www.semanticscholar.org/paper/Effect-of$\underline{\text { Relaxation-Techniques-on-Pain-Associated }}$ MorsyAlam/eafe8a6a3b9ef9d20fdb09823e97bfec2bec1af6 
Oryan, A., Alemzadeh, E.m \& Moshiri, A. (2017). Burn wound healing: Present concepts, treatment strategies and future directions. Journal of Wound Care, 26(1), 5-19.

Park, E., Oh, H., \& Kim, T. (2013). The effects of relaxation breathing on procedural pain and anxiety during burn care. Burns, 39(6), 1101-1106.

Park, J., Shin, S., Kim, J., Song, K., \& Peck, M. (2009). Association between socioeconomic status and burn injury severity. Burns, 35(4), 482-490.

Rafii, F., Mohammadi-Fakhar, F., \& Jamshidi Orak, R. (2014). Effectiveness of jaw relaxation for burn dressing pain: Randomized clinical trial. Pain Management Nursing, 15(4), 845-853.

Tevlin, R., Dillon, L., \& Clover, A., (2017). Education in burns: Lessons from the past and objectives for the future. Burns, 43(6), 1141-1148.

Bozorg-Nejad, M., Azizkhani, H., Ardebili, F. M., Mousavi, S. K., Manafi, F., \& Hosseini, A. F. (2018). The effect of rhythmic breathing on pain of dressing change in patients with burns referred to Ayatollah Mousavi hospital. World journal of plastic surgery, $7(1), 51$. 


\section{تأثثير تمرين التنفس على تقليل الألم المصاحب للغيار بين مرضى الحروق بالمستثفيات}

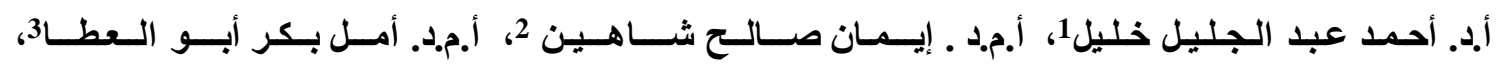

\section{عبيز محمد نصر اللدين يـوسف صـالـ}

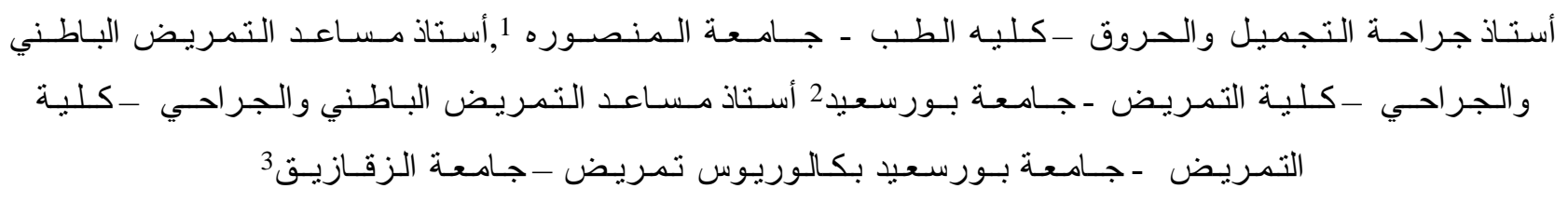

\section{الــــلاصــة}

الخلفية : يعتبر الغيار على الحروق من اهم مسباب الالم لدى مصابى الحروق ـ تـهـف هذه اللاراسـة إلي تقييم تأثير تمرين التتفس على تقليل الألم المصاحب للغيار بين مرضى الحروق بالمستشفيات. العينـة: تضدنت 73 مصاب حروق ـ مكان البحث: مركز الحروق و التجميل بمستشفي جامعة المنصورة . أدوات جمـع البيانـات: مقياس تينيس هده در اسة شبه تجريبية أجريت في

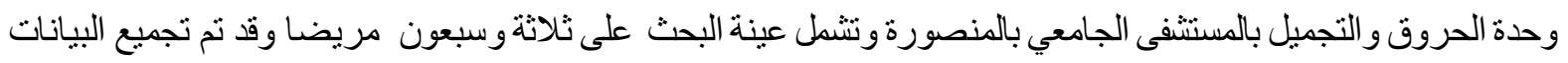

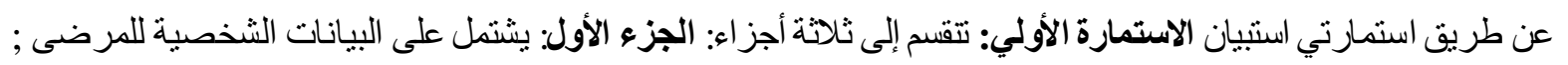

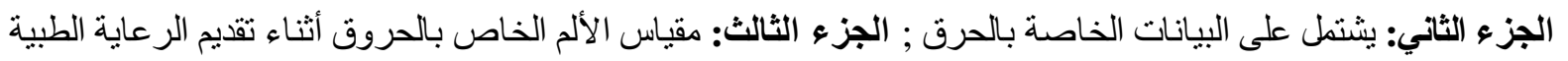

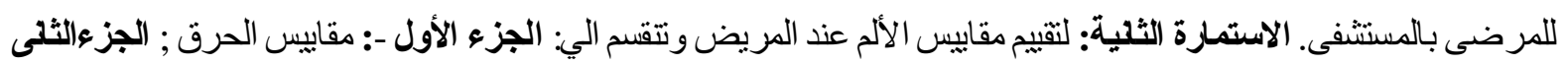

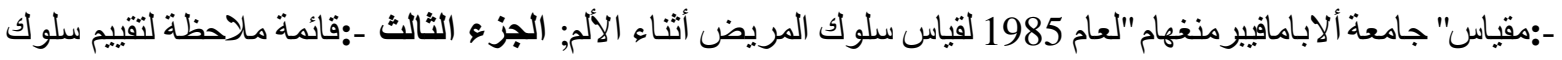
المريض قبل و بعد استخدام تمرين التتفس من خلال ملاحظة سلوك المريض. أوضحت الدر اسة ان هناك تحسن بعد تطبيق ممارسة عملية التنفس فيما يتعلق بمقياس القلق و الألم وبقائمة السلوك ـوقد أثنتت الدراسة ان مقياس الألم انخفض بعد استخدام تمرين التنفس

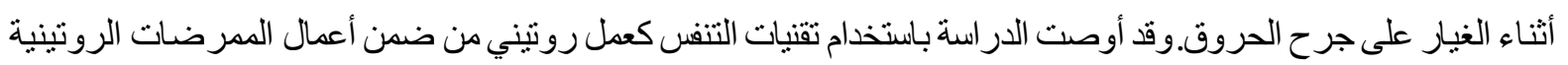
في وحدات الحروق. النتاتج : هناك تحسن فى درجات مقياس الالم و السلوك المصاعب للالم بعد استخدام تمارين التنفس . الخلاصة التوصيات : استخدام تمارين التنفس يقلل الاحساس بالام اثثاء الغيار لذلك يوصى بتعليم المريض تمارين التفس لتقليل الالم المصاحب للغيار

$$
\text { الكلمات المرشدة :تمـرين التنفس, الألــم المصاحب للغيـار ,مـرضى الحـروق }
$$

